\title{
DESARROLLO DE MICROPARTÍCULAS DE QUITOSANO CUATERNIZADO Y ENTRECRUZADO PARA LA ADSORCIÓN DE ÁCIDO DESOXIRRIBONUCLEICO (ADN)
}

\author{
José L. Cconislla Bello*a, Christian Jacinto ${ }^{\text {b }}$, Ily Maza, Martha Jahuira, Alejandra Pando, \\ Holger Mayta, Ana Valderrama
}

\begin{abstract}
RESUMEN
Las micropartículas de quitosano entrecruzado (QE1\%, QE5\%) se prepararon mediante reticulación con el glutaraldehído (GL). Las micropartículas de quitosano cuaternizado (QC) se prepararon mediante cuaternización del grupo amino del quitosano con cloruro de glicidil trimetil amonio (CTAG). Ambas micropartículas de quitosano se caracterizaron utilizando distintas técnicas como la espectroscopía infrarroja con transformadas de Fourier (FTIR), difracción de rayos X (DRX), análisis termogravimétrico (TGA), análisis termogravimétrico diferencial (DTG) y microscopía electrónica de barrido (SEM). La cantidad de ADN adsorbida en las micropartículas se determinó por espectroscopía UV en un equipo NanoDrop2000 obteniéndose resultados satisfactorios. De las isotermas de adsorción evaluadas, el modelo de Freundlich se adapta al proceso de adsorción de ADN.
\end{abstract}

Palabras clave: cuaternización, entrecruzamiento, quitosano, ADN.

\section{DEVELOPMENT OF QUATERNIZED AND CROOSLINKED MICROPARTICLES OF QUITOSANE TO THE DEOXYRIBONUCLEIC ACID (AND) ADSORTION}

\begin{abstract}
The crosslinked quitosane microparticles (QE1\%, QE5\%) were prepared by reticulation with glutaraldehyde (GL). The quaternized quitosane microparticles(QC) were prepared by quaternization of the quitosane amine group with glycidyl trimethyl ammonium (CTAG). The microparticles were characterized and subjected to adsortion tests. To characterization of quitosane microparticles different techniques were used like FTIR spectroscopy, DRX, TGA, DTG and SEM. The DNA quantity adsorbe in the microparticles was determined by uv-visible spectroscopy in a NanoDrop2000 equipment getting very encouraging results. The

\footnotetext{
a Facultad de Ciencias, Escuela de Química, Universidad Nacional de Ingenería, Av. Túpac Amaru 210, Rímac Lima 25, Perú, jcconisllab@uni.com

${ }^{\mathrm{b}}$ Laboratorio de Investigación en Enfermedades Infecciosas, Departamento de Ciencias Celulares y Moleculares, Facultad de Ciencia y Filosofía, Universidad Peruana Cayetano Heredia.
} 
Langmuir and Freundlich adsortion models were applied to describe the balance isotherms. The Freundlich model adapts with the experimental data.

Key words: quaternization, crosslinking, quitosane, DNA.

\section{INTRODUCCIÓN}

Los polímeros naturales, tales como celulosa, agarosa, dextrano y quitosano se han utilizado con frecuencia como material absorbente. El quitosano es un polímero orgánico que existe ampliamente en la naturaleza, es un polisacárido hidrófilo y biocompatible. Sus grupos amino e hidroxilo proporcionan sitios activos de reacción, para el fácil acoplamiento de diversos grupos funcionales e introducir propiedades deseadas. El quitosano natural ha sido modificado por varios métodos (ya sea física o químicamente) con el fin de mejorar la capacidad de adsorción, se han realizado diferentes tipo de reacciones, como por ejemplo formación de policomplejos, de entrecruzamiento y cuaternización (alquilación exhaustiva del grupo amino). ${ }^{1}$

Las muestras de orina se han constituido en una alternativa no invasiva de obtención de ADN para la realización de diagnósticos sensibles y específicos para múltiples enfermedades, dentro de las cuales son de gran interés las infecciosas y parasitarias, entre las que se incluyen: malaria, toxoplasmosis, amebiasis, trichomoniasis, leishmaniasis, tripanosomiasis. Es una técnica muy importante, aunque presenta algunas desventajas debido a que el material genético contenido está presente en bajas concentraciones no detectable por métodos convencionales ${ }^{2}$. La alternativa más plausible es el uso de material polimérico como el quitosano, el cual, debido a la presencia de grupos amino en la cadena polimérica y mediante su modificación química, puede incrementar la eficiencia de sus uniones con el ADN.

En los últimos años se ha demostrado que el quitosano es un potencial portador del gen policatiónico $(\mathrm{ADN})$, este polisacárido biodegradable se une eficazmente al ADN en solución salina o solución de ácido acético protegiéndolo de la degradación por nucleasas. Varios derivados de quitosano han sido preparados en base a las reacciones con los grupos amino libres. Por ejemplo, el quitosano trimetilada se prepara con diferentes grados de cuaternización aumenta la solubilidad del quitosano a pH neutro, el quitosano / ADN Polyplex se sintetiza para la transferencia genética ${ }^{3}$. Todos estos estudios señalan la versatilidad y la promesa del quitosano como un portador de gen $(\mathrm{ADN})$ el cual se ha demostrado.

Se han desarrollado varios modelos de equilibrio para describir la relación de las isotermas de adsorción. Ningún modelo ha demostrado ser aplicable de manera general. Sin embargo, los dos modelos más usados en la literatura son el de Langmuir y el de Freundlich.

El modelo de Langmuir viene representado por la ecuación:

$$
q_{e}=\frac{Q^{\circ} b C_{\theta}}{1+b c_{\theta}}
$$


Donde:

$\mathrm{Q}^{\mathrm{o}}$ : es la concentración de adsorbato por peso unitario de adsorbente que forma una monocapa completa en la superficie, es decir, la capacidad límite de adsorción; y b: una constante del modelo, también llamada coeficiente de adsorción, que se encuentra relacionada con la entalpía de adsorción.

La ecuación (1) se puede linealizar, como se muestra a continuación:

$$
\frac{1}{q_{g}}=\frac{1}{Q^{\circ}}+\frac{1}{b Q^{\circ}} \frac{1}{C_{g}} \ldots \ldots
$$

La ecuación del modelo de Freundlich tiene la forma general:

$$
q_{e}=K_{F} C_{e}^{1 / n}
$$

Donde:

$\mathrm{K}_{\mathrm{F}}$ y $\mathrm{n}$ : son constantes características del sistema. Este es un modelo empírico, aunque se admite que $\mathrm{K}_{\mathrm{F}}$ es una indicación aproximada de la capacidad de adsorción y 1/n: de la intensidad de adsorción.

Los datos experimentales se suelen ajustar usando la fórmula logarítmica de la ecuación (3):

$$
\log q_{e}=\log K_{F}+\frac{1}{n} \log C_{e} \ldots \ldots
$$

De manera que, según la ecuación (4), al representar log qe frente a log Ce, se obtendrá una línea recta de pendiente $1 / \mathrm{n}$ y ordenada en el origen $\log \mathrm{K}_{\mathrm{F}}$.

En este trabajo se busca la preparación y la caracterización de las micropartículas de quitosano modificado: quitosano entrecruzado y quitosano cuaternizado. La importancia de realizar este trabajo se basa en la contribución al desarrollo de preparar posibles micropartículas de quitosano modificado y aplicarlas para la adsorción de ADN y poder aportar en el diagnóstico molecular de enfermedades infecciosas desde muestras no invasivas (orina).

\section{PARTE EXPERIMENTAL}

\section{Métodos de preparación de adsorbentes}

Los tipos de quitosano modificado fueron preparados a partir del quitosano comercial (Grado de Desacetilación (G.D) $=72,49 \%$ y Peso Molecular $(\mathrm{P} . \mathrm{M})=498 \mathrm{KDa}$ ). Para la preparación de QE1\% y QE5\% con GL se disolvieron 2g de quitosano en $150 \mathrm{~mL}$ de ácido acético al $2 \%$ se agregaron distintas proporciones de GL (1\% y 5\%, respectivamente) se agitó hasta obtener un gel y luego se deja reposar por aproximadamente cuatro horas. Seguidamente, se realizan varios lavados con agua ultrapura para neutralizar y eliminar las impurezas, se liofiliza, muele y tamiza (malla $\mathrm{N}^{\circ} 100$ ) para obtener un polvo fino. 
Para la preparación de las micropartículas QC se disolvieron $2 \mathrm{~g}$ de quitosano en $20 \mathrm{~mL}$ de agua ultrapura, se agregó $3 \mathrm{~mL}$ de CTAG bajo agitación a $60{ }^{\circ} \mathrm{C}$ por $24 \mathrm{~h}$. El producto de la reacción fue precipitado en $150 \mathrm{~mL}$ de acetona, luego filtrado y secado a $60{ }^{\circ} \mathrm{C}$. Seguidamente pasa por una molienda y se tamiza con malla $\mathrm{N}^{\mathrm{o}} 100$ para obtener un polvo fino.

\section{Estudio Isoterma de la adsorción de ADN}

Todos los ensayos de adsorción se llevaron a cabo a temperatura de ambiente y se trabajó con ADN plasmídico. Las lecturas de concentración de ADN se determinaron en un equipo NanoDrop 2000, en cada caso se utilizó una determinada masa del adsorbente (3, 6 y $25 \mathrm{mg}$ ) y se puso en contacto con 200, 300 y $500 \mathrm{~mL}$ de solución de ADN a diferentes concentraciones. La adsorción de $\mathrm{ADN}$ se estudió en el rango de 5 a $400 \mathrm{ng} / \mu \mathrm{L}$ a un $\mathrm{pH} \approx 7$, después de mantener en agitación a $350 \mathrm{rpm}$ por $30 \mathrm{~min}$, se centrifugó para separar las dos fases. Se midió la concentración de $\mathrm{ADN}$ presente en la solución remanente.

\section{RESULTADOS Y DISCUSIÓN}

\section{Caracterización de los materiales:}

\section{Análisis e identificación de grupos funcionales por FTIR}

Los grupos funcionales presentes en el quitosano y quitosano modificado (QE1\%, QE5\% y QC) fueron identificados empleando un Espectrofotómetro Infrarrojo con Transformada de Fourier (FTIR) Modelo Shimadzu-1800, en un rango de 4000 a $500 \mathrm{~cm}^{-1}$.

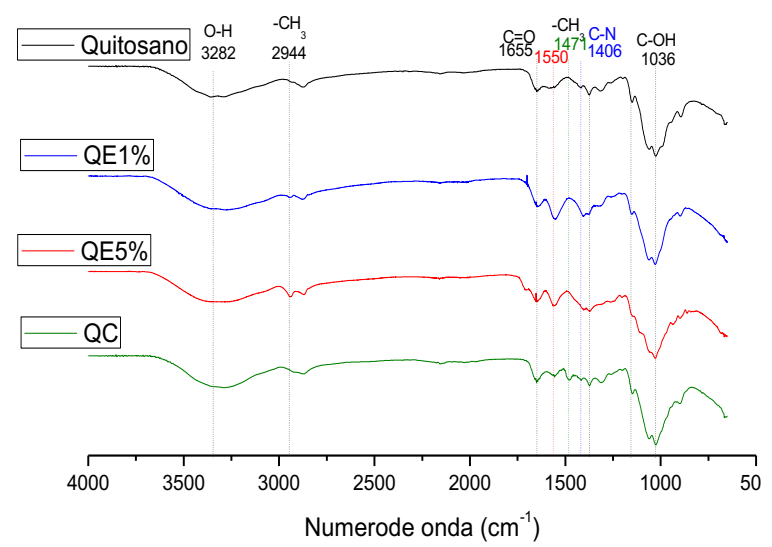

Figura 1. Espectro FTIR de quitosano, QE y QC 
El resultado obtenido es consistente con muchos estudios anteriores ${ }^{7}$, que han demostrado la diferencia en el perfil FITR del quitosano y QE. Los picos de quitosano en $1363 \mathrm{~cm}^{-1}$ y 1155 $\mathrm{cm}^{-1}$ desaparecidos pueden indicar haber sido limitados por el glutaraldehído que retícula al quitosano de igual forma la aparición del pico a $1550 \mathrm{~cm}^{-1}$ puede ser también atribuida al proceso de reticulación. De hecho, Ramachandran et al. ${ }^{8}$ encontraron un nuevo pico agudo a $1610 \mathrm{~cm}^{-1}$ que representa las vibraciones de tensión de C-N en la base de Schiff formada por la reacción de glutaraldehído y quitosano, en nuestro caso ese pico se observa desplazado a $1650 \mathrm{~cm}-1$. Otros autores encontraron esta señal en $1664 \mathrm{~cm}^{-1}$ y $1660 \mathrm{~cm}^{-1}$.

También se observa en la comparación de los espectros FTIR del QC que se evidencia un nuevo pico intenso a $1471 \mathrm{~cm}^{-1}$, probablemente debido a los grupos metilo del amino cuaternario formado por la cuaternizacion del quitosano con CTAG. Estos resultados son consistentes con estudios anteriores ${ }^{10}$. La formación del producto QC, se da por el ataque nucleofílico del grupo amino (-NH2) del quitosano al CTAG (cuaternizante), donde se produce la ruptura del epóxido que forma parte de la estructura del CTAG.

\section{Análisis por DRX}

El quitosano presenta dos formas cristalinas distintas debido a su polimorfismo, los patrones de difracción representan las diferentes mezclas de estas dos formas. Los picos de difracción en el entorno al ángulo $2 \theta=9^{\circ}, 2 \theta=10^{\circ}, 2 \theta=19^{\circ}$ y $2 \theta=20^{\circ}$ observados en el figura 2 son característicos del quitosano y corresponden a resultados publicados anteriormente. ${ }^{11}$

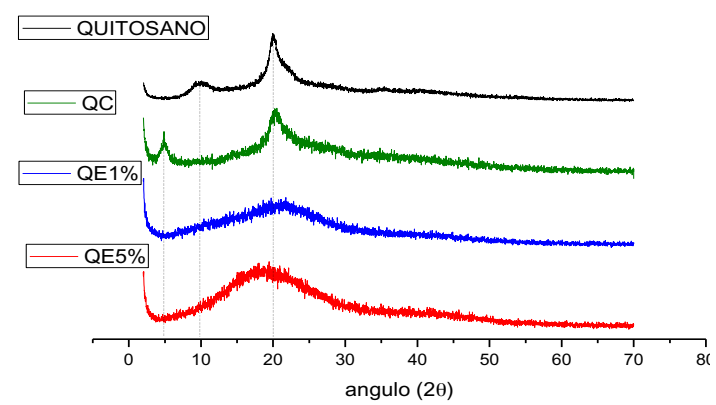

Figura 2. Patrones DRX de quitosano, QE y QC

Al comparar los patrones de difracción entre el quitosano y el quitosano entrecruzado QE1\%, QE5\% se observa picos característicos del quitosano que desaparecen, observando únicamente el pico $2 \theta=21^{\circ}$ algo ensanchado. Esta diferencia corrobora la reacción de entrecruzamiento entre el quitosano y el GL. El amorfismo del pico de QE (QE1\% y QE5\%) pone en evidencia que el índice de cristalinidad del quitosano disminuye después de la reticulación con GL; esto se atribuye a los fuertes enlaces de hidrógeno del grupo amino, los cuales, al ser sustituidos, destruyen eficazmente la regularidad de la estructura del quitosano original, dando como resultado la formación de una estructura amorfa, también se observa que al aumentar la proporción de GL evidenciamos que la intensidad y la anchura del pico están aumentando con lo cual el grado de amorfismo también aumenta ${ }^{11}$. 
Por otro lado, al comparar el patrón de difracción del quitosano y QC, se observan dos picos $2 \theta=5^{\circ}$ y $20^{\circ}$ donde el pico $2 \theta=20^{\circ}$ presenta un ensanchamiento y ya no se observa el pico $2 \theta=9,5$ del quitosano, pero sí un pico nuevo a $2 \theta=5^{\circ}$. La interacción del grupo CTAG con el grupo amino del quitosano originaría una posible disminución del índice de cristalinidad haciendo la estructura más amorfa y la presencia del nuevo pico $2 \theta=5^{\circ}$ en el QC puede ser una evidencia de la formación del grupo amino cuaternario ${ }^{12}$.

\section{Análisis TGA y DTG}

La curva calorimétrica del quitosano (figura 3.a) nos manifiesta tres eventos térmicos. El primer evento está relacionado con la pérdida de humedad que se da entre 60 y $110{ }^{\circ} \mathrm{C}$ debido a la evaporación del agua, el segundo evento podría tratarse de la despolimerización con una máxima pérdida de peso, la cual se manifiesta entre 220 y $300{ }^{\circ} \mathrm{C}$ debido a la degradación de las cadenas principales del quitosano (ruptura de los enlaces C-O-C) además de la separación de grupos acetilo, y el tercer evento podría tratarse de la pirolisis, el cual se da por la descomposición del material orgánico final.
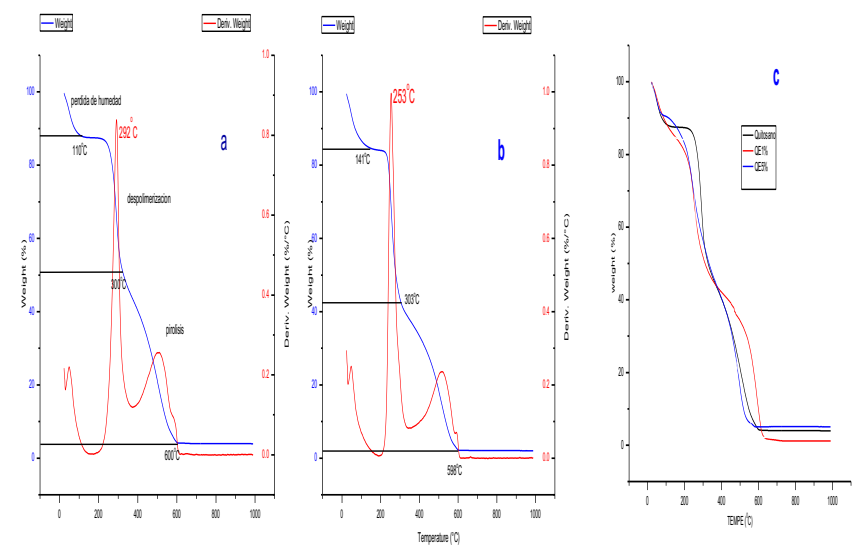

Figura 3. a) TGA y DTG de quitosano b) TGA y DTG QC c) TGA comparativo Q, QC, QE

Los termogramas TGA y DTG del quitosano y el QC (figura 3 a y b) nos muestran una diferencia en su temperatura de máxima velocidad de descomposición $\left(\mathrm{T}_{\max }\right)$. Esta temperatura es mayor para el quitosano en comparación con el QC, probablemente debido a que el quitosano presenta mayor índice de cristalinidad evidenciado en los difractogramas DRX y en los análisis calorimétricos diferenciales de barrido (DTG). También podemos evidenciar que las curvas calorimétricas del quitosano, QE, y QC tienen en común la manifestación de tres eventos térmicos; sin embargo, es el segundo evento el que muestra una diferencia en el valor de Tmax, esto se debe considerar en estos tres polímeros.

Esta diferencia de la cristalinidad se refleja en la estabilidad térmica, puesto que un compuesto, al poseer mayor cristalinidad, su estructura molecular será más rígida y por consiguiente se 
necesita mayor energía para que se pueda descomponer. La disminución en la temperatura de descomposición en el QC se puede atribuir a la disminución en la estabilidad térmica como consecuencia de la interacción de los grupos amino del quitosano. Otra observación se da entre el quitosano y el QE por una diferencia muy notoria en sus termogramas. El QE se observa que es menos estable térmicamente pues inicia con la pérdida de peso alrededor de los $130{ }^{\circ} \mathrm{C}$ dando una descomposición multietapa, en donde los productos intermedios son inestables, esto se puede atribuir al entrecruzamiento de los grupos amino del quitosano y el GL, también la baja cristalinidad del QE es una evidencia más de la baja estabilidad térmica, pues los difractogramas DRX nos muestran que la estructura del QE tiene un grado de cristalinidad muy pobre ${ }^{13}$.

\section{Isotermas de adsorción de ADN}

La figura 4 muestra las isotermas que representan la relación de las capacidades de adsorción de ADN (q) expresado en $\mu \mathrm{g}$ de ADN retenido por gramo de quitosano o quitosano modificado (QE y QC) en agua ultrapura. Teniendo en cuenta las razones de interacción se aprecia que el comportamiento de las isotermas es ascendente hasta una concentración aproximada que varía de acuerdo al tipo de adsorbente (quitosano, QE y QC).

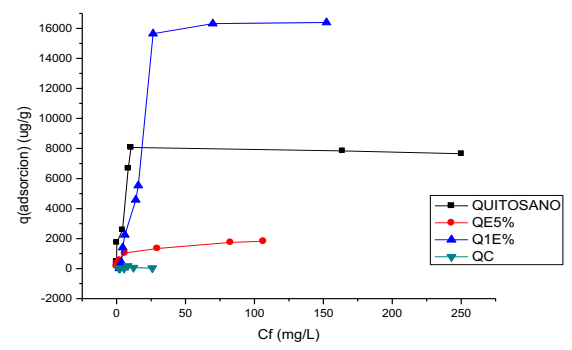

Figura 4. Isotermas de adsorción Quitosano, QE y QC

Los análisis de las muestras para la construcción de las isotermas se hicieron a temperatura de ambiente a un $\mathrm{pH} \approx 7$. Estos resultados muestran que el QE1\% es el que presenta mayor capacidad de adsorción de ADN de toda la serie, con un valor máximo de $\mathrm{q}=15755,3 \mu \mathrm{g} / \mathrm{g}$ (tabla 1). Esta variación en la capacidad de sorción entre QE1\% y QE5\% está relacionada con la variación de la superficie de los sorbentes, es interesante observar que las isotermas para los polímeros (Quitosano, QE1\%, QE5\% y QC) varían considerablemente; de acuerdo con la tabla 1, esto puede ser atribuido a propiedades como el peso molecular y la polidispersidad del polímero de partida. También los QE (QE1\% y QE5\%) pueden tener una mayor porosidad textural que estaría relacionado al contenido de glutaraldehído; por otro lado, los efectos de hidratación contribuyen al sorbente, pues presentan una mayor hinchazón que influirá en la superficie dando una mayor accesibilidad del sorbente hacia los adsorbatos en los dominios de los microporos superficiales y sitios de adsorción accesibles ${ }^{6}$.

Casi todas las isotermas presentan un comportamiento inicial ascendente evidente a excepción de la muestra QC. Inicialmente, se planteó la hipótesis que el QC tendría mayor sorción debido a que es un policomplejo ${ }^{4}$, esto debido a que la interacción ADN-QC se da por orígenes electrostáticos, los cuales son muy sensibles a variaciones de $\mathrm{pH}$ y concentraciones 5 . 
Tabla 1. Valores máximos de q de sorción para quitosano QE y QC.

\begin{tabular}{cc}
\hline Nombre & $\mathrm{q}(\mu \mathrm{g} / \mathrm{g})$ \\
\hline quitosano & 8030,83 \\
\hline QE1\% & 15755,3 \\
\hline QE5\% & 1981,21 \\
\hline QC & 141,61 \\
\hline
\end{tabular}

\section{Modelos de isotermas de adsorción}

La isoterma de adsorción indica cómo las moléculas de adsorbato (ADN) interactúan con el adsorbente (micropartículas de quitosano) en equilibrio en fase líquida como una función de la concentración de adsorbato. En este estudio de equilibrio los datos obtenidos para la adsorción de $\mathrm{ADN}$ en QE fueron los mejores, analizados con los modelos de isotermas de Langmuir y Freundlich.

La forma lineal de la ecuación isoterma de Langmuir se da como:

$$
\frac{1}{q_{\theta}}=\frac{1}{Q^{\circ}}+\frac{1}{b Q^{\circ}} \frac{1}{C_{\theta}} \ldots \ldots
$$

\section{Modelos de isotermas de adsorción}

La isoterma de adsorción indica cómo las moléculas de adsorbato (ADN) interactúan con el adsorbente (micropartículas de quitosano) en equilibrio en fase líquida como una función de la concentración de adsorbato. En este estudio de equilibrio los datos obtenidos para la adsorción de $\mathrm{ADN}$ en QE fueron los mejores, analizados con los modelos de isotermas de Langmuir y Freundlich.

La forma lineal de la ecuación isoterma de Langmuir se da como:

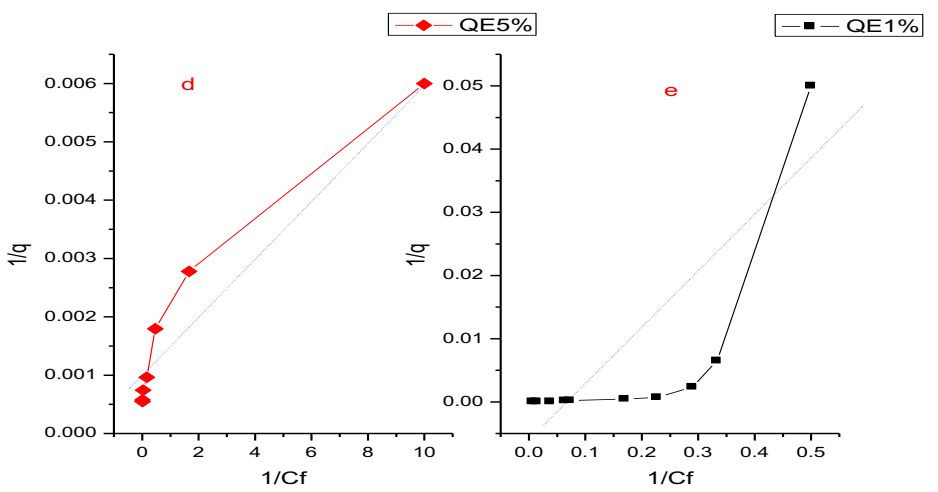

Figura 5. Linealización de Langmuir 
Sin embargo, el modelo de Freundlich se ajustó con mayor eficiencia, como se puede ver en la figura 6. Los datos experimentales cumplen con mayor aproximación con lo predicho por la ecuación (4) la cual describe mejor la adsorción sobre superficies heterogéneas y confirman lo que indicaba la figura 4. Se podría afirmar que no existió influencia de la concentración inicial de sustrato en el sistema estudiado bajo las condiciones de trabajo consideradas, ya que todos los puntos caen aproximadamente sobre una misma recta.

La ecuación de la isoterma de Freundlich se muestra a continuación en su forma lineal:

$$
\log q_{B}=\log K_{F}+\frac{1}{n} \log C_{\theta}
$$

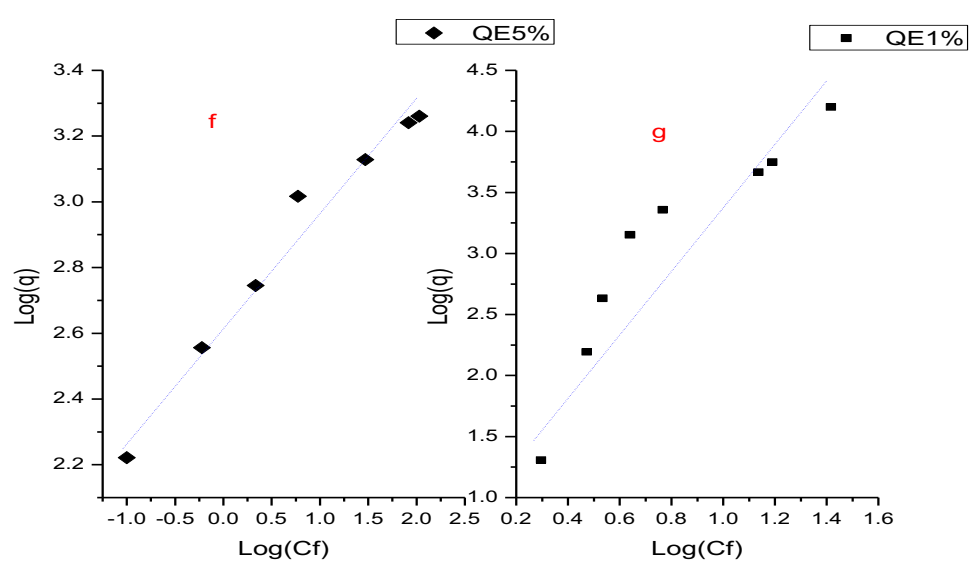

Figura 6. Linealización Freunlich

Tabla 2. Parámetro de los modelo de equilibrio de adsorción de ADN en QE

\begin{tabular}{cccc}
\hline $\begin{array}{c}\text { Modelo de } \\
\text { equilibrio }\end{array}$ & Parámetro & QE1\% & QE5\% \\
\hline Isoterma Langmuir & $\mathrm{b}(\mathrm{L} / \mathrm{mg})$ & 0,05072 & 0,2 \\
& $\mathrm{Q}^{\circ}(\mu \mathrm{g} / \mathrm{g})$ & 1428,57 & 1000 \\
& $\mathrm{r}^{2}$ & 0,6432 & 0,9289 \\
& $\mathrm{R}_{\mathrm{L}}$ & 0,046 & 0,012 \\
\hline Isoterma & $\mathrm{K}_{\mathrm{F}}{ }^{\circ}(\mathrm{L} / \mathrm{g})$ & 51,191 & 421,405 \\
Freundlich & $\mathrm{n}$ & 0,5654 & 2,946 \\
& $\mathrm{r}^{2}$ & 0,8892 & 0,9724 \\
\hline
\end{tabular}


En este estudio del equilibrio de adsorción la tabla 2 nos muestra los datos obtenidos para la adsorción de ADN en micropartículas de QE1\% y QE5\%, considerando los modelos de isotermas de Langmuir y Freundlich.

La isoterma de Langmuir es frecuentemente evaluada por un factor de separación, RL, que se define como sigue:

$$
R_{L}=\frac{1}{1+b c_{0}} \ldots \ldots . .(5)
$$

Donde $\mathrm{C}_{0}$ en este caso es la concentración de soluto inicial más alta. El valor del factor de separación indica el tipo de isoterma y la naturaleza del proceso de adsorción. Considerando el valor $R_{L}$ la adsorción puede ser desfavorables $\left(R_{L}>0\right)$, lineales $\left(R_{L}=1\right)$, favorables $\left(0<R_{L}<1\right)$ o Irreversible $\left(R_{L}=0\right)$. En los resultados observamos que los valores de RL (tabla 2) para QE1\% y QE5\% son favorables, pero los gráficos de linealidad (gráfico 5) son desfavorables.

La isoterma de Freundlich, considerada empírica, describe mejor la adsorción en superficies heterogéneas. Los parámetros de la ecuación linealizada (ecuación 4) donde $\mathrm{K}_{\mathrm{F}}(\mathrm{l} / \mathrm{g})$ es constante de Freundlich y "n" es exponente de Freundlich se determinan a partir de un registro logarítmico de la capacidad de sorción versus logaritmo de la concentración en el equilibrio, Ce, representados en la tabla 2. El valor de $\mathrm{r}^{2}$ nos muestra mejores resultados de linealidad para QE1\% y QE5\% (gráfico 5) y los valores de $\mathrm{K}_{\mathrm{F}}$ son valores no exactos de la capacidad de adsorción.

\section{Efecto del porcentaje de GL en el adsorbente}

Los resultados de los experimentos con diferentes porcentajes de GL en el adsorbente se presentan en la figura 4 (QE1\% y QE5\%), un aumento en el porcentaje de GL en el adsorbente disminuye la capacidad de sorción. La cantidad de adsorbente modificado fue tres miligramos en ambos casos, se trabajó a las mismas condiciones, en QE1\% aumenta la captación de $\mathrm{ADN}$ con respecto al QE5\%. Hay una gran probabilidad que todos los sitios activos sobre la superficie adsorbente estén ocupados y un aumento de la cantidad de GL disminuye la adsorción de ADN.

\section{Microscopía electrónica superficial (SEM)}

La figura 7 presenta las micrografías SEM que ilustra la morfología superficial de las micropartículas Quitosano, QE1\% y QE5\%. En general, este resultado indica claramente que existe una diferencia en la morfología superficial entre quitosano y QE. De hecho, en comparación con la morfología suave, densa y plana del quitosano la del QE tiene una superficie porosa y poco áspera (figura $1(\mathrm{QE} 1 \%$ y QE5\%)). Estos resultados deben atribuirse al entrecruzamiento y al grado de desacetilación que son los que influyen en el tamaño y la morfología superficial del QE, todo esto es consistente con estudios anteriores ${ }^{14}$.

De igual forma, se puede observar la diferencia en la morfología superficial QE1\% y QE5\%, debido al porcentaje de entrecruzamiento con glutaraldehído, este entrecruzante puede aumentar la rugosidad de la superficie de QE aumentando el porcentaje de glutaraldehído, 
lo que indica que la reacción ha tenido lugar en la superficie. Además, la estructura porosa de QE puede ofrecer más sitios de adsorción para adsorbatos, que generalmente apoyan el hecho que el quitosano entrecruzado con glutaraldehído ha sido ampliamente estudiado en la absorción de metales pesados ${ }^{15}$. Además, QE 5\% con una superficie muy rugosa y una estructura de poros más abiertos podría suponer que es favorable para interactuar con el ADN a cierto punto, debido a que el mayor entrecruzamiento y la rugosidad de la superficie pueden evitar la interacción del adsorbato con el adsorbente, lo que puede explicarse con los resultados de las isotermas de adsorción.

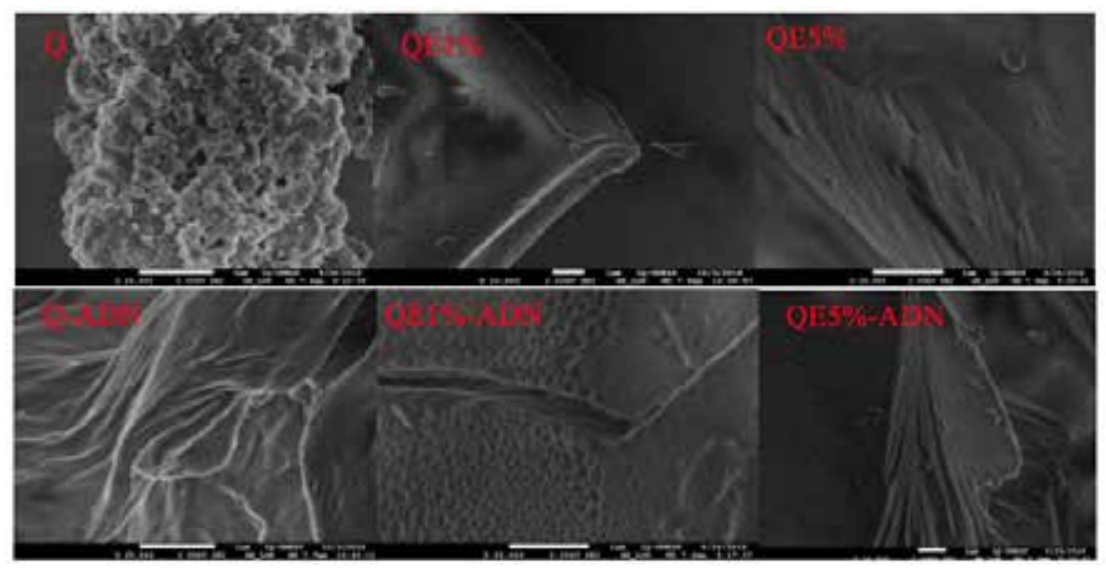

Figura 7. Imágenes SEM comparativas micropartículas de quitosano-ADN, QE1\%-ADN y QE5\%-ADN, aumento x 10000 y x25000.

En la figura se compara las micropartículas quitosano, QE1\% y QE5\% con y sin ADN, se puede evidenciar que la morfología superficial de las micropartículas sufren cambios, una posible evidencia de que el ADN está siendo adsorbido por estas en la superficie. También se observa en la imagen SEM de quitosano-ADN que el cambio de la morfología es muy evidente, una posible explicación a esto es que los grupos amino del quitosano interactúan con el ADN, aglomerando las micropartículas de quitosano. En QE1\%-ADN también se puede evidenciar el cambio en la morfología superficial evidenciando la posible adsorción de $\mathrm{ADN}$, esto puede estar generando una mayor rugosidad de la superficie inicial de QE1\%, lo mismo se evidencia para QE5\%-ADN.

\section{CONCLUSIONES}

Se prepararon diferentes tipos de quitosano y quitosano modificados (QE1\%, QE5\%, y QC) partiendo de quitosano comercial, los cuales se caracterizaron por diferentes técnicas FTIR, DRX, TGA, DTG y SEM, estos resultados fueron consistente con la literatura.

Las pruebas de adsorción demostraron que las micropartículas de QE1\% adsorben el ADN en mayor cantidad. Considerando la capacidad de sorción de 15,76 mg ADN/g QE 1\% El proceso de adsorción de ADN se aproxima al modelo de isoterma de Freunlich, el cual describe mejor la sorción en superficies heterogéneas. 


\title{
AGRADECIMIENTO
}

\author{
INNOVATE PERU-118-PNICP-PIAP-2015.
}

\section{REFERENCIAS BIBLIOGRÁFICAS}

1. Yazdani-Pedram M, Retuert J, Quijada R. Hydrogels based on modified chitosan, 1. Synthesis and swelling behavior of poly(acrylic acid) grafted chitosan. Macromol Chem Phys. 2000;201(9):923-930.

2. Spanos A, Theocharis G, Karageorgopoulos DE, Peppas G, Fouskakis D, Falagas ME. Surveillance of community outbreaks of respiratory tract infections based on house-call visits in the metropolitan area of Athens, Greece. PLoS One. 2012;7(8):e40310. doi: 10.1371/journal.pone. 0040310 .

3. Layek B, Singh J. Chitosan for DNA and gene therapy. Chitosan Based Biomaterials. 2013; 2: 209-244.

4. Bordi F, Chronopoulou L, Palocci C, Bomboi F, Di Martino A, Cifani N, et al. ChitosanDNA complexes: Effect of molecular parameters on the efficiency of delivery. Colloids Surface A. 2014;460:184-90.

5. Oskolkov NN, Potemkin II. Complexation in asymmetric solutions of oppositely charged polyelectrolytes: Phase diagram. Macromolecules. 2007; 40(23):8423-8429.

6. Wilson LD, Mohamed MH, Headley JV. Surface area and pore structure properties of urethane-based copolymers containing $\beta$-cyclodextrin. J Colloid Interface Sci. 2011;357(1):215-222.

7. Yao W, Jiao Y, Luo J, Du M, Zong L. Practical synthesis and characterization of mannose-modified chitosan. Int J Biol Macromol. 2012;50(3):821-825.

8. Ramachandran S, Nandhakumar S, Dhanaraju MD. Formulation and characterization of glutaraldehyde cross-linked chitosan biodegradable microspheres loaded with famotidine. Trop J Pharm Res. 2011;10:309-316.

9. Knaul JZ, Hudson SM, Creber KAM. Crosslinking of chitosan fibers with dialdehydes: Proposal of a new reaction mechanism. J Polym Sci Pol Phys. 1999;37:1079-1094.

10. Zhang W, Sun C, Zhao Y, Lu X. One-pot synthesis and characterization of crosslinked quaternized chitosan microspheres as protein adsorbent. Int J Biol Macromol. 2011;49(4):688-92.

11. Souza BWS, Cerqueira MA, Martins JT, Casariego A, Teixeira JA, Vicente AA. Influence of electric fields on the structure of chitosan edible coatings. Food Hydrocolloid. 2010;24(4):330-5.

12. Tang R, Zhang Y, Zhang Y, Yu Z. Synthesis and characterization of chitosan based dye containing quaternary ammonium group. Carbohyd Polym. 2016;139:191-6.

13. Corazzari I, Nistico R, Turci F, Faga MG, Franzoso F, Tabasso S, et al. Advanced physico-chemical characterization of chitosan by means of TGA coupled on-line with FTIR and GCMS: Thermal degradation and water adsorption capacity. Polym Degrad Stabil. 2015; 112:1-9. 
14. Mirzaei BE, Ramazani SAA, Shafiee M, Danaei M. Studies on glutaraldehyde crosslinked chitosan hydrogel properties for drug delivery systems. Int J Polym Mater. 2013; 62:605-611.

15. Suguna M, Kumar NS, Reddy AS, Boddu VM, Krishnaiah A. Biosorption of lead (II) from aqueous solution on glutaraldehyde cross-linked chitosan beads. Can J Chem Eng. 2011;89:833-843. 